\title{
Un dispositivo grupal como herramienta para la adscripción al sistema de salud ${ }^{1}$
}

\section{A group device as a tool for affiliation to the health system}

\author{
Carlos Gabriel Colusso ${ }^{2}$
}

\section{Resumen}

El presente artículo tiene por objetivo comunicar resultados, lecturas y reflexiones producidas en el análisis de un caso de inclusión de dispositivos grupales alternativos a las lógicas manicomiales, en el campo de la Atención Primaria de la Salud.

En primer término, consta de una descripción del espacio, sus orígenes, problemáticas a las que da respuesta, y finalidades, de acuerdo a la mirada de los entrevistados. Incluye en este punto algunos fundamentos teóricos del dispositivo, lo que da ocasión a pensarlo en relación a las tecnologías blandas y el trabajo vivo en salud.

Los apartados siguientes se centran en la indagación de los procesos de coordinación asistencial: el primero, en lo referido a la planificación, y su imbricación con las instancias de gestión; el siguiente intenta ubicar la coordinación expresada en transiciones -como trayectos de los usuarios entre los procesos de atención singular y el espacio colectivo, y como producción de espacios transicionales en los que se superponen proyectos terapéuticos individuales y del dispositivo-; mientras que el último consiste en una digresión, necesaria, relativa a intersecciones entre tecnologías, arreglos institucionales, y dispositivos.

Las consideraciones finales, incluyen una reflexión acerca del lugar de lo micropolítico en las condiciones de posibilidad de producción de salud.

Palabras clave: Coordinación asistencial Adscripción- Dispositivo - Trabajo vivo Cogestión.

\section{Summary}

The objective of this article is to communicate results, readings and reflections produced along the analysis of a case of inclusion through group devices alternative to psychiatric logics, in the Primary Health Care Area. In the beginning, we describe the space, its origins, problems to which it responds and purposes, according to the interviewees'opin-

\footnotetext{
${ }^{1}$ El presente artículo se basa en el Trabajo Integrador Final de la Carrera de Especialización en Psicología Clínica, Institucional y Comunitaria denominado "Un caso de inclusión de dispositivos grupales alternativos a las lógicas manicomiales, en el campo de la Atención Primaria de la Salud”, de mi autoría, dirigido por Mgtr. Adriana Huerta, presentado en 2019.

${ }^{2}$ Psicólogo, Especialista en Psicología Clínica, Institucional y Comunitaria (UNR). Trabaja en la Dirección de Centros de Salud de la Secretaría de Salud Pública de la Municipalidad de Rosario.
} 
ions. It includes some theoretical basis which gives occasion to think it in relation to soft technologies and live work in health. The following sections focus on the investigation of the processes of aid coordination: the first, in terms of planning and its interweaving with the management; the next one tries to locate the coordination expressed in transitions -as paths for users- between the processes of singular attention and the collective space, as a production of transitional spaces in which individual and device therapeutic projects overlap; the latter consists of a digression, necessary, relating to intersections between technologies, institutional arrangements and devices. The final considerations include a reflection about micropolitical and the conditions of possibility of health production.

Keywords: Care coordination - AdscriptionDevice - Living work - Co-management.

\section{Presentación}

El presente artículo busca exponer los resultados de una indagación sobre un dispositivo grupal que funciona, desde 2014, en un centro de atención primaria de la salud (CAPS) dependiente de la Municipalidad de Rosario; y se ha centrado en el análisis de procesos de coordinación entre ambas instancias, durante el año 2018.

Se trata de un espacio grupal del que participan jóvenes y adultos, muchos de los cuales atraviesan graves situaciones de sufrimiento subjetivo, con consecuencias en sus posibilidades de establecimiento de lazos.

De acuerdo a lo enunciado por sus coordinadores, si bien se considera un espacio abierto a la comunidad, es activamente ofrecido a personas con diversas problemáticas vinculadas a la salud mental, y a quienes su padecimiento de distintos grados de estigmatización y discriminación las sitúan en grave posición de vulnerabilidad, al articularse en sus historias vitales aquellas sanciones y prácticas relacionadas con las representaciones tradicionales de la enfermedad mental, y la pertenencia a sectores sociales afectados por acentuados procesos de desafiliación. Así, la población a la que se dirige este espacio se puede caracterizar como un grupo excluido o incluido de manera frágil, que no establece lazos que resulten significativos, sin proyectos que permitan proyectarse en algún futuro diferente, $\mathrm{y}$ con historias singulares signadas por la pobreza, la discriminación, las prácticas de encierro, el aislamiento, la violencia.

El espacio tiene como finalidad la apropiación subjetivante de objetos culturales, y la construcción de nuevos lazos; la ocupación de espacios vitales inéditos a partir del intercambio y el compartir lo producido (interpretación de canciones, dramatizaciones, dibujos) en el grupo, en actividades abiertas a la comunidad y en otros eventos, albergando la diversidad, y sin afectar la libertad individual.

En el marco del trabajo aquí presentado, la experiencia fue indagada como caso de inclusión de dispositivos grupales alternativos a las lógicas manicomiales en los centros de salud que forman parte de sistemas de salud basados en los principios de la Atención Primaria de la Salud (APS).

Como particularidad, este trabajo intentó abordar una caracterización desde la perspectiva de la organización de los servicios de salud, analizando la relación entre el dispositivo y el CAPS. Para ese análisis, se tomó como eje principal a los procesos de coordinación asistencial entre ambas instancias, sus grados de realización, vacilaciones e impasses.

La categoría de coordinación asistencial, alude a los procesos de ajustes y concertaciones entre niveles, servicios, y otras instancias de atención dirigidas a la integración de las res- 
puestas asistenciales. En los sistemas de salud, los problemas de coordinación entre niveles asistenciales se manifiestan fundamentalmente en la ineficiencia en la gestión de recursos, pérdida de continuidad en los procesos, y disminución de la calidad de la atención prestada (Terraza Núñez, Vargas Lorenzo y Vázquez Navarrete, 2006). Dado que esos problemas toman mayor relevancia en pacientes crónicos, resultó pertinente indagar estos procesos de coordinación asistencial en relación a la situación delimitada como objeto de la indagación, donde un horizonte de cronicidad de los padecimientos subjetivos asoma en muchos casos singulares.

Este trabajo se desarrolló en un contexto que ubicaba a la experiencia analizada bajo la influencia de movimientos sociales vinculados a la defensa de los derechos de usuarios de servicios de salud mental, y del fuerte desarrollo que la APS ha tenido en la ciudad de Rosario desde los años noventa, ambos procesos en sintonía con la promulgación y defensa de la Ley $\mathrm{N}^{\circ} 26657$.

La construcción de una Ley Nacional de Salud Mental y Adicciones centrada en los derechos humanos fue el resultado de un proceso del que han participado colectivos de usuarios, familiares, profesionales, asociaciones gremiales. Su implementación requiere de una red de servicios territorializados que garanticen el acceso a derechos, y favorezcan los procesos de externación e inclusión social de personas con padecimiento mental, tendiendo, además, a evitar nuevas internaciones.

En ese sentido la inclusión de los problemas de salud mental en la estrategia de APS resulta fundamental para la construcción de espacios asistenciales y prácticas contextualizadas con perspectiva comunitaria, en tanto la afectación de la vida social es marca presente en los casos de padecimientos graves, y teniendo en cuenta que los efectos de la in- justicia social configuran particularidades en los modos de producción de subjetividad y formas del sufrimiento.

En el ámbito de la salud pública local, en torno a la situación de los dispositivos grupales se configura una preocupación común a muchos trabajadores, ligada a la legitimación de estos espacios al interior de las mismas instituciones desde las cuales se desarrollan, frente a la población, y ante los actores que representan instancias centrales de gestión. En términos generales, puede sostenerse que esa preocupación responde a que "los dispositivos y prácticas no tradicionales persisten más como iniciativas de compromiso individual o experiencias extraordinarias que como parte de las políticas públicas en el sector" (Zaldúa, Pawlowicz, Tisera, Longo, Sopransi, Lenta, Moschella y Lohigorry, 2014).

Esta problemática de legitimación, que a veces parece colorear la cultura institucional sin encontrar instancias de tramitación adecuada, ha tomado un tinte de gravedad en el contexto nacional durante los últimos años, donde las políticas más generales y las referidas a la Salud Pública se han presentado como amenazas y retrocesos concretos respecto del desarrollo de modelos de atención centrados en el paradigma de derechos.

Resulta, por ello, de gran importancia registrar, analizar y comunicar experiencias que se consideren solidarias a este modelo.

El análisis al que se alude a continuación se realizó desde una perspectiva de estudio de caso, cualitativa, exploratoria, tomando inspiración en algunas consideraciones referidas al método indiciario.

La información fue relevada a partir de instancias de observación participante, entrevistas con trabajadores ${ }^{3}$, y el acceso a docu-

\footnotetext{
${ }^{3}$ Se ha entrevistado a la jefa del CAPS, la trabajadora social, dos médicos generalistas, una de ellos, además, coordinadora del espacio, y a una antropóloga que
} 
mentos de gestión de las Direcciones de Centros de Salud y de Salud Mental, e informes, notas y proyectos realizados por los integrantes del dispositivo.

\section{Caracterización del dispositivo}

\section{Sobre los orígenes}

En las entrevistas, los relatos relacionados a las motivaciones del primer armado del dispositivo, no hicieron mención al cumplimiento de leyes o de mandatos institucionales, sino la inquietud del equipo por construir formas de abordaje creativas a problemas que no podían ser alojados suficientemente desde una organización donde las intervenciones se producían exclusivamente a través de dispositivos de consultas individuales.

De acuerdo a esos relatos el momento de creación del espacio, en 2014, apareció coincidente con la conformación de un "nuevo equipo" -así fue denominado por diferentes actores indagados- en el CAPS, integrado en mayor parte por nuevos trabajadores. Los entrevistados concordaron al contar que a fines de 2013, la violencia y la hostilidad habían crecido de tal modo en el territorio, que llevaron a la mayoría de los anteriores trabajadores a sentirse amenazados y efectuar traslados a otros puestos.

Se presentó en el equipo, entonces, una inquietud vinculada a la sustentabilidad de los procesos de trabajo, articulada a la necesidad de volver a alojar a los usuarios del CAPS. Se consideró que para que el territorio -signado por la presencia de violencia, hostilidad, crueldad- fuera habitable para el equipo del CAPS, había que trabajar haciendo habitable

también coordina el espacio grupal, pero no forma parte del staff del CAPS. el espacio del centro para los usuarios que viven en el territorio.

Puede decirse que el origen del dispositivo parecía ligarse a las estrategias que "el equipo nuevo" fue construyendo en esos momentos $^{4}$, tanto para dar respuesta a problemas poblacionales, como para producir una habitabilidad del territorio que parecía perdida.

La búsqueda y el desarrollo de metodologías adecuadas para dar alojamiento y tramitación a diferentes problemas de salud de la población son intrínsecos a la APS como estrategia. Este planteo incluye la dimensión ética de la organización del trabajo, ya que las distintas formas de asistencia se expresan diferentes modos de lazo social (Galende,1998). Esto resulta de particular relevancia en el caso, ya que esa organización del trabajo condiciona las posibles respuestas e intervenciones de los equipos de salud, en procesos de saludenfermedad-atención dados en un contexto signado por la inequidad, la vulnerabilidad, la desafiliación.

El espacio grupal nació como una instancia originalmente pensada por trabajadores del CAPS en relación a problemáticas de sus usuarios, como creación en un clima de cierta frescura dada por la conformación de un nuevo equipo - con cierta distancia a posibles procesos de mortificación en lo instituidoque se propuso establecer modos de trabajo que incluyan una lectura del territorio.

Una de las condiciones fundamentales para la posibilidad de una coordinación entre instancias asistenciales, está dada por una comunidad de estrategias y objetivos (Vargas,

\footnotetext{
${ }^{4}$ Cabe señalar que esas nuevas estrategias construidas por trabajadores recién llegados se apuntalaron en el trabajo conjunto con algunos - pocos-integrantes del equipo anterior, como la psicóloga que continuó en el CAPS durante un año más. Su presencia fue importante para dar continuidad a lazos entre los usuarios y la institución que, evidentemente, no estaban radicalmente rotos.
} 
Vázquez, Henao y Campos, 2009). De acuerdo a lo planteado hasta aquí, podría señalarse que esa condición se encontraba allanada en los inicios de la experiencia.

Para profundizar sobre esta afirmación y confrontarla con la realidad del espacio durante el período aquí abordado -2018-, se ubicarán las problemáticas de los usuarios, las finalidades del espacio y el tipo de tecnologías desplegadas, de acuerdo a lo señalado por los trabajadores.

\section{Problemas de salud de los usuarios}

Si un problema se constituye en la distancia entre una realidad observada por un actor social y un valor o deseo referido a cómo debería ser (Rovere, 1993), puede enunciarse que la brecha entre una necesidad en salud y su satisfacción sería una forma posible de expresión de los problemas de salud.

Se caracterizará a continuación la noción necesidades en salud, como un modo de aproximación a la descripción de problemas de salud, en tanto la construcción discursiva de una necesidad implica justamente una idea referida a un valor o deseo disímil con la realidad observada.

Cecilio (2009) teoriza sobre las necesidades en salud, señalando que el equipo de salud debe trabajar para despejarlas ya que, por lo general, aparecen en la consulta, bajo el modo de demandas más o menos inespecíficas y de manera individual. Con la finalidad de describir y operacionalizar el concepto, propone una taxonomía de cuatro categorías: 1) condiciones de vida; 2) acceso a las tecnologías de salud capaces de mejorar y prolongar la vida; 3) vínculos -efectivos y afectivos- entre cada usuario y un equipo o profesional; y 4) grados de autonomía de cada persona en sus modos de conducir su vida, en relación a la lucha por la satisfacción de sus necesidades.

Las cuatro categorías son aplicables a la construcción de los problemas de salud de la población con la que el dispositivo intentaba trabajar, y las dimensiones a las que aluden pueden hallarse en los dichos y enunciados de los trabajadores.

$\mathrm{Si}$ bien en las entrevistas aparecieron aproximaciones a la descripción de los problemas de los usuarios desde la mención de categorías diagnósticas - "trastorno de salud mental", "retraso mental", "problemáticas de consumo", "psicosis"- en general se hizo hincapié en cuestiones ligadas a las dificultades para la vida social, leídas desde los modos de transitar la institución.

Se habló de personas que no tenían espacios de socialización, o tenían escasamente y en general, reducidos a lo familiar. Se pueden ubicar dos modos en los que el aislamiento apareció como problemática: por un lado, aquellos que prácticamente no salían de sus casas; por otro, quienes pasaban mucho tiempo en el centro de salud, circulando sin ninguna actividad, sin pedidos consistentes o claros, cuestión que comenzó a leerse en clave de demanda.

Los trabajadores ubicaron las problemáticas de estos usuarios en torno a la posibilidad de establecer lazos con otros y vivir con autonomía. Como veremos en seguida, también identificaron la necesidad de acceso a tecnologías adecuadas a la problemática, frente a la cual este espacio grupal apareció como respuesta. Más adelante se intentará pesquisar qué calidad de vínculos terapéuticos se han construido a nivel singular desde el espacio, como también si se han producido lecturas y consecuentes intervenciones en relación a las condiciones de vida, de alcance intersectorial. 


\section{Finalidades}

En las entrevistas, las finalidades del espacio aparecieron muy ligadas a la caracterización anterior. Fueron señaladas: la constitución de una grupalidad, el establecimiento de lazos de amistad y amorosos, y -cuestión subrayada por todos los entrevistados- el aumento de autonomía de los participantes.

Además de las finalidades ligadas directamente a la salud de los usuarios, han mencionado otras, que referían a dar respuesta, desde los nuevos modos de trabajo, a problemas organizacionales provocados por ciertas manifestaciones - "demandas inespecíficas", para el discurso médico- de los usuarios.

El médico entrevistado relató que algunos usuarios del dispositivo aparecían como "policonsultantes", reconociendo, además, que las respuestas a sus demandas, en la consulta, no eran efectivas en términos de satisfacción, o calma del malestar.

Al respecto cabe ensayar la siguiente lectura: en el consultorio, se produce un trabajo de significación de la demanda desde el saber médico. Bajo el imperio de prácticas médicohegemónicas, y en el contexto dado por una organización que propone soluciones estandarizadas a ocasiones disímiles, se da respuesta al sufrimiento desde un saber que, en su imbricación con el poder, puede resultar en un acallamiento del sujeto. Como conjetura, puede pensarse que, en esta situación, un malestar que aparece con un intento de ciframiento en el cuerpo, no logra constituirse como mensaje, e insiste, produciendo una situación de "polidemanda".

Esa insistencia motivó un cuestionamiento a la eficacia de la modalidad de respuesta practicada en el consultorio, dando lugar en el equipo a la idea de producción de otro alojamiento de esa demanda, que implicara ubicar un paréntesis en lo referido a su satisfacción. Pensar en una "adscripción diferente" -como señalara una entrevistadadio lugar a la inclusión de una perspectiva de equidad; de respuestas específicas, diferenciadas, a problemáticas de salud que afectan de distintos modos a distintos sectores o miembros de esa población.

Retomando las ideas de Cecilio (2009), puede decirse que el dispositivo se constituyó como forma de hacer accesibles para un grupo de usuarios tecnologías adecuadas a sus necesidades.

\section{Pensando el espacio grupal como tecno- logía}

Desde la declaración de Alma-Ata (OMS, 1978), tomó relevancia como tema de salud pública la cuestión de las tecnologías apropiadas para la APS, con diferentes propuestas o desarrollos de acuerdo a la concepciones de APS en las que encontraran sustento.

Desde un punto de vista técnico, la tecnología consiste en la organización de los recursos que se destinan al cumplimiento de una determinada tarea; y como término, refiere a los procesos de trabajo (Testa, 1988).

Merhy, Feuerwerker y Burg Ceccim (2006) desarrollan una diferenciación dentro de las tecnologías en salud. Denominan "blandas" a aquellas que reposan en el trabajo sobre la relación con el paciente, aspectos transferenciales, atravesamientos institucionales, poder. Estas tecnologías posibilitan al trabajador actuar sobre las realidades singulares de cada usuario en cada contexto, utilizando las tecnologías duras y blanda-duras como una referencia. Para los autores, en el espacio privado en que ocurre la relación intersubjetiva entre trabajador y usuario, es posible la construcción de una libertad de hacer las cosas de manera que produzcan sentido, al menos para 
los trabajadores, pero preferentemente para ambas partes. La expresión "trabajo vivo" alude al trabajo creativo en el cual los trabajadores de salud reinventan constantemente su autonomía en la producción de actos de salud.

El trabajo dirigido a propiciar autonomía en los usuarios será efectivo según las posibilidades de los trabajadores de ejercer su propia autonomía en las relaciones terapéuticas que se instituyan, y de que puedan sostener una posición emancipada frente a mandatos que hagan hegemónicos otros sentidos de la institución. Es decir, que logren transitar por las tensiones que supone el alojamiento de lo singular en espacios públicos, y el auspicio de una autonomía en usuarios marcados por el estigma de la incapacidad y el desvalimiento.

A la vez, la posibilidad del trabajo vivo está relacionada a la disposición de los recursos para cumplir con la tarea.

Esa organización de los recursos requiere de otro trabajo, de gestión, con una planificación adecuada a las finalidades.

En algunas de las entrevistas, pudo leerse el planteo de "lo grupal" o "el arte" como valores en sí mismos, con algún componente genuino que hace de por sí a cierta producción de subjetividad. Sin embargo, pudo apreciarse que la lógica del dispositivo no se agotaba en formas y contenidos dados, sino que ponía en juego una estrategia que implicaba "la oferta de lazo social", a través de un encuadre particular planteado por los coordinadores, en un tiempo y lugar determinados. Un encuadre, a la vez, siempre puesto en cuestión, a partir del ejercicio de una constante interrogación a la luz del tipo de lazos que allí se establecieran.

Al mismo tiempo, el alojamiento singular en el dispositivo, la posición adoptada en relación al lazo, implicaba necesariamente algún movimiento del sujeto, siendo función del equipo hacer lectura del mismo, sosteniéndolo a veces o interrogándolo otras.
En relación a la delimitación conceptual de un espacio denominado "dispositivo grupal alternativo a las lógicas manicomiales", que intenta producir "modos de adscripción diferentes", se situarán algunas ideas respecto de los términos de su denominación.

En referencia al concepto de dispositivo, Agamben (2011) retoma los desarrollos de Foucault, resumiendo en tres puntos sus elementos principales:

1) se trata de un conjunto heterogéneo que incluye virtualmente cada cosa, sea discursiva o no: discursos, instituciones, edificios, leyes, medidas policíacas, proposiciones filosóficas. El dispositivo, tomado en sí mismo, es la red que se tiende entre estos elementos. 2) El dispositivo siempre tiene una función estratégica concreta, que siempre está inscrita en una relación de poder. 3) Como tal, el dispositivo resulta del cruzamiento de relaciones de poder y de saber. (p. 250)

De acuerdo a sus afirmaciones, un dispositivo siempre implica procesos de subjetivación: "Llamo dispositivo a todo aquello que tiene, de una manera u otra, la capacidad de capturar, orientar, determinar, interceptar, modelar, controlar y asegurar los gestos, las conductas, las opiniones y los discursos de los seres vivos" (Agambem, 2011, p. 257).

La configuración de un dispositivo tendrá determinaciones sociales, históricas, culturales. En ese sentido, un CAPS es un espacio institucional con múltiples atravesamientos, y puede verse como una coreografía compleja en la que coexisten distintos dispositivos, que a su vez soportan distintos modos de producción de subjetividad.

Respecto de la característica grupal del espacio, será condición para sostener la vitalidad 
del trabajo estar advertidos de la complejidad que lo grupal implica. Siguiendo a Percia (1997),

Para que un grupo pueda instituir sus acciones como propias, es necesario un trabajo crítico sobre su dispositivo de producción. Se quiere decir, una crítica que revele como problema el poder productor de los dispositivos grupales (p. 45)

Percia sostiene que no debe pensarse en alguna esencialidad que defina lo grupal ya que "el grupo es la producción de un espacio común, en el que se realiza una implicación diferente" (p. 43). Se puede pensar, entonces, el grupo como un momento en lo colectivo, en el cual hay convergencia respecto del tema o motivo, pero la manera de afectación es singular. "El deseo puede coincidir sobre una misma figura, pero no se ajusta según una forma general de articulación" (p. 43).

De acuerdo a lo planteado, el valor terapéutico del espacio, su potencialidad en tal sentido, puede ubicarse en relación, al menos, a dos dimensiones. Una de ellas se vincula a la condición subjetivante del dispositivo, al ofertar al sujeto lugares a ocupar en el lazo; alude a la ética en tanto esa oferta requiere del análisis crítico del dispositivo fundante de lo grupal. La otra refiere a la clínica de la singularidad, en tanto es posible allí una lectura de las afectaciones en juego frente al encuentro con aquellos lugares ofrecidos, y del sostén y otras intervenciones adecuadas a ese nivel singular.

Por su parte, la adjetivación de alternativo respecto de los modos de producción de subjetividad esperables, hace referencia al lugar del saber y el poder. Es así que alternativo refiere a la diferenciación y distanciamiento con el modelo manicomial.

El dispositivo, en tanto tiene una función que siempre está inscrita en una relación de poder, y resulta del cruzamiento de relaciones de poder y de saber, no se reduce al espacio grupal, con sus días y horarios de encuentro, sino que se extiende a todo el reticulado relacional que hace posible su existencia.

Como ya se ha señalado, su sostenimiento en relación a organizaciones del trabajo más clásicas (tayloristas), y modos de pensar las prácticas vigentes (hegemonías disciplinares), requiere de otras tecnologías, de gestión, $y$ planificación, que permitan su desarrollo.

\section{Procesos de coordinación en la planifi- cación, gesta, y gestión del dispositivo}

La integración de las áreas de dirección y apoyo a las estrategias es una condición para que sea posible una adecuada coordinación asistencial (Vargas et al. 2009).

Esa integración puede cobrar existencia en espacios formales de planificación, en la gestión de recursos, pero también puede darse a leer en la cotidianeidad de los lugares de trabajo, en la toma de decisiones con autonomía e interacciones "laterales" que requiere un espacio de trabajo con alto nivel de incertidumbre.

Resulta importante situar algunos planteos de Onocko (2002) acerca de la cogestión. Hablando de la gestión como productora de intersubjetividad y procesos transicionales, sostiene que, entre la heterogestión taylorista y la utopía autogestionaria, la vida se desenvuelve en constante cogestión. Así, la revaloriza como dimensión de creación de la gestión, en tanto la función que operarían allí los gestores, o jefes, sería la de acompañar procesos creativos entre trabajadores dando lugar a relaciones intersubjetivas al modo de procesos transicionales (Winnicot, 2007).

Los distintos tipos de cogestión pueden considerarse como modalidades de la inte- 
gración directiva o gerencial. Según Onocko (2002), el primer momento de la planificación de un proyecto, muchas veces es la estructuración de un grupo como intersubjetividad, para lo cual los jefes deben garantizar un tiempo y un lugar, y ofrecer suficientemente sostén y manejo, "estando" jefes, y no "siendo" jefes (p.6).

En las entrevistas se manifestó un reconocimiento del apoyo de la Dirección de Salud Mental en lo relativo a la conformación de un espacio central y seguimiento de los espacios grupales locales, con gestiones de recursos humanos y materiales. También apareció ubicado un aporte económico para compra de materiales desde la Dirección de Centros de Salud; dándose por descontados los recursos humanos y espacios físicos destinados ${ }^{5}$. Sin embargo, se mencionó la ausencia de espacios de planificación que pusieran en relación sistematizada los procesos de gestión local con el dispositivo. De acuerdo a lo señalado, los encuentros entre equipo de coordinación y trabajadores con funciones formales de gestión de la Secretaría de Salud han sido muy puntuales a lo largo de los años de funcionamiento del dispositivo, y han estado dirigidos a resolver cuestiones de recursos de manera acotada.

Varios entrevistados se detuvieron en el mayor o menor lugar del dispositivo como tema en la agenda de las reuniones de equipo del CAPS. Las opiniones fueron disímiles, y sostenidas también en diferentes modos de pensar el ingreso del tema en esa agenda; al-

\footnotetext{
${ }^{5}$ El equipo de coordinación del espacio, en 2018, estaba conformado por: una médica generalista y un psicólogo integrantes del equipo del CAPS, una psicóloga que desarrollaba sus prácticas en el marco de una carrera de especialización de la UNR, una antropóloga que dependía administrativamente de la Dirección de Salud Mental, y una profesora de teatro que se sumó a partir de la articulación con el Programa Nueva Oportunidad, en el marco de un proyecto de capacitación de promotores culturales.
}

gunos pensaban que era suficiente informar sobre el trabajo realizado, mientras otros problematizaban en relación a la dificultad de hacer de esas reuniones un momento de planificación. Frente a la interrogación acerca de esa dificultad, aparecían como respuestas problemas de organización, la bastedad de temas y problemas a tratar, y la priorización de problemáticas por su carácter de urgencia. Este era un tema que podría esperar. También se ha señalado en las entrevistas que, a la vez, en los espacios de retrabajo de los coordinadores del dispositivo no participaban otros integrantes del equipo de salud, ni se constituían otros espacios sistemáticos para la discusión de casos.

$\mathrm{Al}$ interior de la Coordinación del dispositivo, parecía reflejarse algo de esa insuficiencia: quienes coordinaban el espacio sin formar parte del equipo del CAPS, no percibían sostener relaciones con otros trabajadores de ese equipo. Así, la lectura de los procesos y sucesos que transcurrían en el dispositivo quedaba bastante alejada del conocimiento de otros aspectos de los procesos de atención de los participantes.

Tras el análisis de las entrevistas y otras fuentes, ha quedado enunciada una interrogación acerca de la relación entre la falta de definición del tiempo y lugar necesarios para que la cogestión se produzca, y otros desencuentros en niveles centrales de gestión de los que aquella dificultad podría ser correlato.

En consonancia con la escasez de instancias de cogestión, en el equipo de coordinación aparecieron dichos que transmitían una crítica a lo que podría leerse como una precariedad ligada a la ausencia de apuntalamientos sostenidos desde instancias de gestión de mayor centralidad, instituidas y reconocidas. Frente a esto señalaron un reaseguro puesto en el apoyo político de la Dirección de Salud Mental al desarrollo de los espacios grupales 
que, a la vez, fue reconocido como frágil, y sujeto a posibles cambios de acuerdo al signo político de futuros gobiernos.

Si bien la coordinación del espacio reconoció como problemática la precariedad en los apoyos, en tanto insuficiencia de la cogestión, esto parecía por momentos resolverse en un deslizamiento hacia el sostenimiento de una ilusión de autogestión, de suficiencia. Esta idea se hacía manifiesta en la ausencia de invitaciones, por parte del equipo de coordinación, a otros trabajadores del CAPS a participar de instancias de planificación.

A lo largo de las entrevistas, se han omitido las temáticas de capacitación e instancias de supervisión, omisión que también puede leerse como correlato de esa idea de "autosuficiencia”. Este punto se pone de relieve teniendo en cuenta que en las observaciones participantes, se advirtió que las reuniones del equipo de coordinación ocurrían en un clima de mucha intensidad y ritmo de trabajo, con grandes flujos de información, toma de decisiones y definición de estrategias apoyadas en lecturas clínicas.

En un sentido diferente del de los planteos hasta aquí recuperados, los dichos de otra coordinadora del espacio abonaron la idea de que había, quizás invisibilizado, un trabajo a nivel de la gestión local; en tanto desde el entorno laboral del espacio y sus coordinadores se mantenían condiciones mínimas necesarias, aquellas pequeñas cosas que tienen que suceder todos los días para que una actividad se desarrolle sin inconvenientes mayores: el orden y la limpieza del lugar, la buena recepción a los participantes, el trato amable aun cuando la actividad se volviera ruidosa y desordenada.

Podemos conjeturar que los integrantes de los equipos sostenían y producían interacciones "laterales", con acuerdos y resolución de problemas sin intervención directa de directivos o gestores, que conducían a un nivel de legitimación de la experiencia al interior del equipo de salud, produciendo participaciones indirectas, en el cuidado del espacio de trabajo o del tiempo que destinaba a él la coordinadora médica, por ejemplo.

En general, podría sostenerse que estos niveles de participación del resto del equipo de salud se encontraban relacionados a una aceptación del valor del dispositivo como espacio de producción de salud.

Los entrevistados subrayaron el respeto del equipo de salud hacia el tiempo que sus compañeros dedicaban al espacio, en especial, el destinado por la coordinadora que además cumplía funciones como médica. Este hecho implicaba una reducción de la oferta asistencial bajo la forma de consultas, y complicaba la organización de la respuesta a demandas "espontáneas, del día”, en especial las sostenidas por personas ya referenciadas a esa médica. De acuerdo a distintos fragmentos de entrevistas, el sostenimiento del tiempo dedicado a prácticas alternativas a las tradicionales no se produjo sin dificultades, como un reflejo de la tensión en torno a la legitimación del espacio.

En la figura de la médica/coordinadora de taller de arte, quien respondía o maniobraba entre demandas de distinta índole, se manifestaban, de manera condensada y desplaza$\mathrm{da}$, los conflictos que las distintas prácticas -y los sentidos que instituyen- gestan en la institución.

\section{Transiciones, puentes entre la aten- ción singular y el espacio grupal}

\section{Derivación e inclusión de usuarios en el dispositivo}

Respecto de las modalidades de inclusión de los usuarios en el dispositivo, los relatos 
apuntaron a que inicialmente fueron convocados por la médica y la psicóloga que iniciaron el espacio. Al momento del relevamiento, se partía de la invitación o sugerencia de distintos trabajadores, médicos, psicólogos, trabajadora social, enfermeros, que comentaban previamente la situación a los coordinadores del espacio, en general a la médica y el psicólogo que integraban, también, el equipo del centro. Asimismo, algunos usuarios llegaban por invitación de otros participantes.

Entre los criterios y estrategias para la derivación de usuarios al dispositivo, podemos señalar que para algunos trabajadores se mantuvieron los criterios iniciales en relación a las dificultades de socialización.

Otros manifestaron que se habían producido ampliaciones de criterios para la derivación al espacio, cambios en relación a la ruptura de barreras que hacían pensar que el espacio era exclusivamente para "pacientes que tienen trastornos de salud mental". Se invitaba "ahora" a personas sobre las que se consideraba que necesitaban recreación, espacios de encuentros con otros y que brindaran herramientas de expresión artística.

Las maneras en que la oferta se presentaba eran variadas, e iban desde ofrecer la participación como una propuesta que formara parte del proyecto terapéutico, hasta acercamientos de apariencia más informal y/o espontánea, invitando al "club de amigos".

Como obstáculo para que algunas derivaciones se hicieran efectivas, se mencionaron los prejuicios y posiciones discriminatorias frente a la visibilidad del padecimiento mental, que reducían el acercamiento al espacio de nuevas personas. Frente a esto, se encontraron dos posiciones. La primera, mantenía en los enunciados una partición de los seres humanos entre aquellos que portan enfermedades o trastornos mentales y los que no, planteando que alguna gente invitada al espa- cio no accedía ya que "piensan que es exclusivamente para personas que tienen trastornos de salud mental". La segunda posición no desconocía los efectos de la estigmatización, pero no ubicaba las consecuencias de la discriminación sobre la convocatoria al espacio como un obstáculo, sino como límite de la estrategia.

\section{Trayectos desde el dispositivo a espacios singulares}

Entre las modalidades de transición desde el dispositivo a los espacios singulares, puede señalarse que el momento de desarrollo de la actividad, era una oportunidad para visibilizar y enunciar problemas y dolencias de distinta índole, desde las más puntuales como una cefalea u odontalgia, a otras de mayor impacto en la historia vital y que requerían abordajes más complejos, como una situación de violencia familiar, o la necesidad de construir la elección de un método anticonceptivo.

En los primeros casos, estos emergentes eran tratados a partir de la derivación del usuario al espacio asistencial (consultorio de enfermería, médico, odontológico) más accesible, de acuerdo a la organización que disponía ese día el CAPS para resolver la "demanda espontánea".

Frente a las otras situaciones, se trataba de coordinar un trabajo con el médico de referencia y otros integrantes del equipo, de acuerdo a una primera ponderación de la pertinencia de un abordaje interdisciplinario.

Tal como fue señalado en los relatos, y pudo ser observado, los usuarios se acompañaban en el movimiento del espacio grupal a la atención singular, una vez definida la forma en que ésta se ofrecía.

Un punto llamativo era la tendencia de los usuarios a elegir a la coordinadora del espa- 
cio como médica para la atención singular, condicionados, tal vez, por la construcción de lazos de confianza que allí se producían. En las observaciones se pudo advertir el grado de implicación de los usuarios con el dispositivo en tanto lugar para decir: hablaban de sus experiencias cotidianas, sentimientos, disfrutes, dificultades; hablaban de sí mismos, con esa implicación con la propia palabra que tiene por condición la presencia de un otro/Otro, una escucha. Sostener ese lugar era una de las funciones de los coordinadores, también de la médica. Se producía, entonces, un efecto de cierta horizontalización de las relaciones de poder, en tanto el saber que se desplegaba y tenía preponderancia era el saber sobre sí, en permanente develamiento.

Esta situación fortalecía y facilitaba procesos de adscripción, pero también los concentraba en trabajadores con los cuales los usuarios establecían relaciones en el dispositivo.

Es importante también señalar que, además de que los coordinadores se constituían en ocasiones como agentes derivadores - para que fueran atendidas cuestiones que los usuarios vivenciaban como necesidad de atención-, los usuarios se apropiaban del espacio del CAPS logrando una confianza en la relación con la institución desde la cual se habilitaban a realizar pedidos y consultas sin requerir un acompañamiento directo, y de manera efectiva. Incluso se produjeron pedidos encadenados (la demanda de uno permitía a otro ubicar allí un derecho o la posibilidad de un beneficio para sî).

\section{Coordinación de los proyectos terapéuti- cos singulares y grupales}

Desde el enfoque de mayor pragmatismo, la presencia frecuente de estos usuarios en el CAPS facilitaba el seguimiento. Por ejemplo en el caso de aquel que debía realizar controles de salud que incluían extracciones de sangre frecuentes, la enfermera iba a su encuentro cuando él asistía al dispositivo.

Se ha señalado que el espacio, en tanto dispositivo, se presentaba como una forma de “adscripción diferente". Puede sostenerse que una adscripción lograda, con un cierto grado de realización supone el establecimiento de proyectos terapéuticos singulares.

Un nivel aceptable de coordinación entre los espacios - centro de salud y dispositivo grupal- debía mantener una coherencia de propósitos y métodos, que se manifestara en los procesos y recorridos singulares de los usuarios por estos ámbitos de salud.

En algunos casos, quizás las coincidencias generales en torno a objetivos de ambos espacios, sostenidos a la vez por trabajadores sujetos a los mismos proyectos generales y lineamientos, fueran suficiente garantía para que aquella coherencia se sostuviera. No obstante, en otras situaciones, la evaluación de procesos de atención enmarcados en diversas planificaciones, requería de mecanismos de facilitación.

En consonancia, la decisión ligada a proponer que un usuario asistiera al espacio grupal, y transitara por el dispositivo como parte del proyecto terapéutico singular, requería del establecimiento de ciertos mojones y orientaciones del trabajo, producidos en una lectura compartida del caso.

Por tal motivo se relevó la existencia de espacios de retrabajo de los casos, y las instancias de articulación entre el equipo del dispositivo y los equipos de referencia de los usuarios.

De acuerdo a las entrevistas, el equipo de coordinación del dispositivo trabajaba en la lectura de los procesos singulares tal como tenían ocurrencia en el marco colectivo, pensando y redefiniendo en esa clave intervencio- 
nes y modalidades de coordinación.

Por su parte, el CAPS establecía distintos modos de encuentro entre los trabajadores implicados en la reflexión sobre un caso: reuniones de equipo, reunión de casos complejos, espacios más informales, "de pasillo"; y reuniones interdisciplinarias agendadas de manera puntual en relación a una situación. Sin embargo, no ha habido encuentros de trabajo clínico entre el equipo de coordinación del espacio grupal y el equipo de referencia de un usuario, según lo referido en las entrevistas.

Ese trabajo quedaba a cuenta de la médica y el psicólogo que integraban ambos equipos, y encarnaban la intersección entre ambos grupos de trabajo. Esta solución de compromiso aparecía como una salida posible frente a dificultades ya descriptas, pero iba en desmedro de un trabajo interdisciplinario y entre equipos (coordinación del dispositivo y equipo de referencia) de mayor riqueza.

El trabajo conjunto sobre los proyectos terapéuticos debería posibilitar la configuración de distintos tipos de transiciones entre el CAPS y su cúmulo de ofertas asistenciales, por un lado, y el dispositivo, por otro.

Estas transiciones, además de ser señaladas como recorridos de personas de un espacio a otro, pueden pensarse como espacios transicionales, donde el proyecto terapéutico singular exista en el transcurrir del espacio colectivo, produciéndose esa existencia en zonas superpuestas (relacionadas con el devenir del sujeto y del espacio colectivo).

Las operaciones sobre el encuadre, la proposición de determinadas consignas de trabajo para el "taller", u otras intervenciones orientadas por la lectura de los procesos singulares, fueron produciendo recortes de esas zonas donde se superponen la experiencia colectiva y el desarrollo del proyecto terapéutico singular. Ejemplo de ello son los señala- mientos dirigidos a ubicar las agresiones entre usuarios, suscitadas por dificultades de un usuario para estar alojado en el espacio; la disposición del espacio al auspicio del encuentro de otro usuario y su "potencial" acompañante terapéutico; o el trabajo sostenido en el proceso de ubicar la anticoncepción como tema de salud. Todo esto señala la producción de recortes con implicaciones singulares, y el trazado de trayectos particulares que permiten el acceso a prácticas relacionadas a la salud.

\section{El lugar de lo intersectorial. Perspecti- vas de integralidad}

Otro punto abordado es el de los posibles impactos de los procesos de coordinación entre el dispositivo y la asistencia a nivel singular, en el desarrollo de la intersectorialidad en los proyectos terapéuticos.

Considerar "la salud como la capacidad singular y colectiva para luchar contra las condiciones que limitan la vida" (Ferrandini, 2011, p.1) implica trabajar desde una concepción amplia de los procesos de salud-enfermedad-atención, considerando relaciones de determinación y condicionamiento que van desde lo microbiológico a las políticas sociales, teniendo en cuenta que, tal como señala Sávio Alves (2009), no toda la oferta que requiere la atención de los problemas de salud complejos es inherente al sector salud.

De acuerdo a lo relevado, la participación del dispositivo permitía al equipo una lectura más precisa de condiciones de vida de los usuarios, y su traducción en necesidades de salud. Además, el tránsito de los usuarios por el espacio grupal, allanaba el camino para trabajar en otras intervenciones.

La coordinación con la trabajadora social y otros integrantes del equipo para el acompañamiento de la gestión de beneficios sociales 
(certificados de discapacidad, pase de transporte, pensiones), derivó en una línea de articulación con las áreas estatales que trabajan con personas con discapacidad, con buenos resultados. Este fue un punto central de las prácticas de asistencia social en relación a los usuarios del dispositivo.

En las entrevistas se resaltó positivamente la articulación con un aula radial, dependiente de una escuela primaria para adultos, que funcionaba en el espacio físico del CAPS y a la cual asistían varios de los usuarios. Se valoró también el rol activo y de mutuo acompañamiento entre participantes para llegar a ese espacio y sostener la asistencia. La participación del espacio grupal y el andamiaje construido, fueron considerados fundamentales para la reinclusión de varios usuarios en el sistema educativo. Asimismo, se encontraron vínculos con Economía Social para el apoyo de proyectos puntuales de emprendimientos productivos de los cuales algunos usuarios han participado, con resultados satisfactorios para ellos. No obstante, la inclusión laboral y el acceso a la vivienda han sido señaladas, en distintas entrevistas, como las articulaciones menos logradas.

La articulación con otros espacios y propuestas terapéuticas que se inscriben en el mismo paradigma ideológico político en relación a la defensa de los derechos humanos y la sustitución del manicomio, fue planteada como un recorrido de particular importancia en el desarrollo y fortalecimiento de la experiencia. Las instancias de encuentro con dispositivos sustitutivos como El Puente, El Gomecito, Pomelo en el Patio, Café con Leche, han propiciado la apertura de trayectos y circuitos de intercambio para los usuarios; como también favorecieron la construcción de una identidad grupal, mediada por el encuentro con otros. Para los coordinadores, estas relaciones también se constituyeron en apoyo e intercambio frente a problemáticas comunes.

\section{Pensando el espacio grupal como dis- positivo}

Los dispositivos "buscan subvertir las líneas de poder instituidas. Un dispositivo sólo podrá ser confirmado en esta función, a posteriori" (Onocko, 2002, p.2).

Se describirán aquí aquellos aportes del espacio grupal que -de acuerdo al reconocimiento de los entrevistados- para el equipo de salud propiciaron la interrogación de prácticas instituidas.

En primer lugar, se señalaron aportes a la discusión del equipo de salud, que produjeron reflexiones y una mayor sensibilidad de sus integrantes respecto de la estigmatización relacionada al sufrimiento psíquico y sus consecuencias en el establecimiento de lazos, así como en lo relacionado a perspectivas terapéuticas solidarias a la APS. También se ubicó una valoración positiva de los espacios colectivos $-\mathrm{y}$ su relación con el arte, el encuentro con otros, el disfrute de distintas actividadescomo alternativas terapéuticas, que impactó en la colaboración del equipo para que el espacio se sostuviera.

Además, se manifestó que el dispositivo producía un impacto en la práctica médica. En primer término, proponía una mirada crítica de las relaciones entre profesionales y usuarios a las que no podría calificarse de "vínculos terapéuticos" cuando producen o sostienen objetalización y asimetrías. El segundo alcance sobre la práctica médica, se relacionaba con facilitar el desarrollo de una "ampliación del objeto de la clínica" (Souza Campos, 2009), en tanto el trabajo con los usuarios aportaba lecturas acerca de cómo las condiciones de vida y los modos de posicionarse en el lazo social de los sujetos forman parte de los procesos de salud-enfermedad.

Por último, y como principal aporte a la interrogación de prácticas instituidas, frente 
a demandas amplias, indefinidas, que no encontraban adecuada tramitación en saberes previamente concebidos (se producía en todo caso una insistencia, repetición, a veces desbordes de la organización de la asistencia), el equipo encontró otra modalidad de abordaje. Poniendo en suspenso las respuestas, y a la vez alojando a los sujetos en escenas de interlocución, dando a esas demandas un estatuto de significante, tratando de catalizar procesos tendientes a la producción subjetiva ligada a la historización, a la enunciación a nombre propio, y a una apertura a la dimensión performativa del lenguaje; construyendo modos de intervención desde el espacio colectivo, orientadas al establecimiento de modos de lazo posibles para los usuarios. Por esta vía se produjo una promoción de posiciones singulares y autonomía. Este proceso impactó a favor del seguimiento y la relación del usuario con los distintos trabajadores del CAPS, y las propuestas terapéuticas que ellos sostenían.

"A posteriori", en tanto se dirija a subvertir líneas de poder instituidas, quizás el espacio grupal pueda ser confirmado como dispositivo, sólo por momentos, atentos a lo que se produce fugazmente.

Como continuidad, en cambio, tal vez sea conveniente considerarlo un arreglo institucional, con especificidad de dominio sobre algunos espacios y prácticas. Para Onocko (2002), a los arreglos institucionales los caracteriza:

cierta estructuración y permanencia, en ese sentido pretendemos formas de organización que puedan ser institucionalizadas. La máquina de producir control no opera por pulsaciones intermitentes, opera como flujo continuo. Por eso, hemos trabajado buscando desarrollar arreglos que detenten la potencialidad de producir esos flujos en la dirección contraria. (p. 2)
Puede, entonces, buscarse al dispositivo en las vertientes de las tecnologías blandas de las que abrevan los arreglos institucionales.

En tanto el espacio grupal sostenía una función estratégica concreta, a la que podría llamarse hacer lugar a lo que desborda, había una disposición de medios orientada a tal fin, relacionada a la posibilidad de mayor movilidad en las relaciones de poder y saber. Ello requirió de la apelación a herramientas tecnológicas: el desarrollo de encuadres, técnicas grupales, recursos artísticos. Pero además de estas tecnologías, hacer lugar requiere de prácticas con vocación instituyente, prácticas no obedientes. Hacer lugar es hacérselo a la singularidad, en la hiancia de la institución en tanto "consagración de un sentido que excluye las diferencias que el estilo instituye" (Jinkis, 1983, p.17).

Como ejemplo de esa vocación, puede tomarse las palabras de Ulloa (2011) sobre su relación al dispositivo analítico: "un psicoanalista no tiene un proyecto, ni siquiera un buen proyecto, que defina cómo debe 'curarse' un paciente, sino que su papel es asumir la dirección de la cura sin marcar una dirección" (p.99).

Si la vocación en el dispositivo se vale de direcciones, será a fin de conmover la dirección consagrada, a fin de hacer lugar al estilo.

"No se tratará aquí de hablar del arte, sino de hacerle caso, de soportar sus consecuencias" (Ariel, 1994, p.15).

\section{Consideraciones finales}

Para una recapitulación de lo desarrollado hasta aquí, se han tomado como ordenadores cinco condiciones para que la coordinación asistencial sea posible: políticas públicas con recursos suficientes y continuidad en el tiempo, de las que la estrategia forme parte; la 
existencia de objetivos y estrategias comunes entre las instancias; un modelo organizativo que se base en el primer nivel de atención y sus equipos como encargados de integrar y coordinar los procesos de atención; la integración de funciones de dirección o apoyo a las estrategias; y la disposición de los trabajadores al uso de mecanismos de coordinación (Vargas, et al.,2010, p 33-35).

En relación al primer punto, si bien las políticas de salud nacionales en 2018 fueron adversas para el desarrollo de planificaciones sostenidas en prácticas de salud mental fundadas en los principios de la APS, el contexto local propició condiciones más favorables a la realización de proyectos como el dispositivo analizado. Es importante considerar el clima de época, que recorría las instituciones públicas, en especial aquellas vinculadas al sector de la salud mental, en donde la defensa del paradigma de derechos humanos, la resistencia a la avanzada de políticas neoliberales en la transformación del sistema de salud, y la oposición a la adecuación de leyes y normativas en respuesta a intereses corporativos aparecen como eje articulador de un colectivo. En términos amplios, los direccionamientos de las políticas públicas a nivel provincial y municipal se han encontrado en consonancia o, al menos, condicionados por esos procesos. Esto ha permitido el desarrollo de dispositivos alternativos a la lógica manicomial y al modelo médico-hegemónico, y en algunos casos como el analizado, su crecimiento en articulación con otros sectores, como la Universidad Nacional de Rosario, y programas de Desarrollo Social provincial.

Como un elemento fundamental, debemos señalar el apoyo a los dispositivos sustitutivos que el Ministerio de Salud provincial ha llevado adelante, ya que su reconocimiento institucional a partir de decretos, la creación de un área de coordinación administrativa, y la asignación de un presupuesto, supusieron un avance en la consolidación de estas estrategias en el ámbito provincial.

Asimismo, directamente ligado a esta experiencia, debe repararse en el rol de la Dirección de Salud Mental municipal. Como acciones concretas en dirección al sostenimiento del dispositivo, esta dirección ha dado continuidad a un espacio de encuentro denominado "Dispositivos Grupales en la estrategia de Atención Primaria”, cuyo objetivo principal fue construir herramientas que visibilicen y den legitimidad a estos espacios. Se trata de una intención dirigida a las estructuras de gestión y administración, a todo el colectivo de trabajadores, y a la población en general. Este espacio se constituyó en un lugar de reflexión donde poner a trabajar dificultades de la práctica.

También ha propiciado la gestión de recursos humanos y materiales junto a la Dirección de Centros de Salud, desde la cual se ha sostenido a lo largo de los años la disposición de tiempo de trabajadores del CAPS, y de un espacio físico.

Estas valoraciones positivas referidas al medio local se presentan en contraposición a las políticas nacionales, sin desconocer las problemáticas desplegadas en el desarrollo del trabajo, los señalamientos de los entrevistados dirigidos a la fragilidad de estas condiciones y a la endeblez de la inscripción institucional en la política de salud. Tanto las estimaciones acerca de la responsabilidad de la Dirección de Centros de Salud en la insuficiencia de procesos de cogestión, como los modos precarios de contrataciones y asignaciones de presupuestos, sumados a la consideración de una atadura de los lineamientos en políticas de Salud Mental a la continuidad de actuales gestores, han configurado un horizonte con la presente amenaza hacia la continuidad de los dispositivos alternativos que se desarrollan en centros de salud. 
El segundo eje refiere a los objetivos y estrategias en común entre el CAPS y el dispositivo. Respecto de ello, se ha encontrado una coherencia, que parece estar en relación a la coincidencia temporal de los orígenes del espacio grupal, y un particular momento de la historia del CAPS. Dado que se trataba de un equipo de salud en reemplazo de trabajadores que debieron trasladarse por problemas de violencia en el territorio, se hizo imperioso realizar un diagnóstico del lazo social, y de las problemáticas

A las condiciones de producción de lazo social propias de la época y de los sectores poblacionales más afectados por la injusticia social, debe agregarse la particular presencia del narcotráfico, que impuso para esta población nuevas implicancias, no sólo económicas, sino también en el marco de significaciones e identidades sociales: modos de goce, acceso al consumo, relación con lo prohibido, representaciones de la vida y la muerte, la ética y la figura del semejante.

En ese contexto el equipo del CAPS puso particular atención en construir estrategias de alojamiento que configuraran una habitabilidad del espacio. El dispositivo no parece nacer ajeno a esta problemática, sino como un desprendimiento de ella, construyendo una propuesta diferenciada para ese alojamiento desde un criterio de equidad. Esta propuesta se ordena en relación al trabajo sobre el lazo social, fundamentalmente en el punto en el cual para algunas subjetividades, y frente al debilitamiento del tejido social, se vuelve necesaria una función de amparo.

En el contexto territorial, la propuesta aparece adecuada como alternativa de tratamiento para padecimientos subjetivos, pero alcanza a una problemática poblacional más generalizada.

Sobre la tercera condición, referida a la existencia de un modelo organizativo que se base en el primer nivel de atención y sus equipos, como encargados de integrar y coordinar los procesos de atención, claramente esa intencionalidad aparece en la descripción de los procesos de trabajo. De hecho en las entrevistas se afirma que en términos de accesibilidad y seguimiento este "modo de adscripción particular" allana el camino para las prácticas disciplinares a las que los usuarios acceden, no sólo facilitando el contacto, sino también proporcionando a los profesionales alguna lectura previa de las problemáticas que baliza las intervenciones.

Si bien el CAPS propone métodos para la adscripción que incluyen la asignación de un médico de referencia para los pacientes, el vínculo que los usuarios y los coordinadores del dispositivo construyen, en ocasiones lleva a la superposición de funciones en el direccionamiento de los proyectos terapéuticos, y a que los integrantes del equipo del dispositivo cumplan algunas funciones de coordinación de la atención en la red que en otros casos ocupan los médicos de referencia. También sucede que algunos usuarios comienzan a atenderse con la médica que coordina el dispositivo, dejando a sus médicos de referencia asignados. ¿Debería verse este tipo de situaciones como un problema? En el caso de pensar que son consecuencia de construcciones de vínculos propias de este tipo de dispositivos, y que hacen a una mayor calidad de atención, desde una perspectiva de mayor integralidad y con una distribución más democrática del poder y el saber, la respuesta sería que no. No obstante, podría contestarse que sí, en caso de ubicar que esos vínculos no constituyen aportes para procesos direccionados a una transformación más global de las prácticas al interior del equipo de salud, produciendo heterogeneidad y fraccionamiento en las ofertas de atención. La respuesta también debería ser afirmativa si esos modos del lazo 
llevaran a una concentración del desarrollo de distintas dimensiones de la vida, al modo de la institución total; esto no aparece como una dificultad en el caso, y su mención funciona aquí a modo de balizamiento.

Ya se ha indicado una insuficiencia en la construcción de un tiempo y un lugar para la elaboración conjunta de los casos y proyectos terapéuticos. Esta insuficiencia lo es también para dirimir acuerdos sobre modos de hacer las prácticas, entre los equipos de referencia del centro y los coordinadores del espacio.

Ciertos argumentos organizacionales (Vargas et. al., 2010), llevan a señalar aquellas situaciones donde la planificación de las prácticas no puede sostenerse en una centralidad, ni en la normalización con instrumentos como guías o protocolos, dado que el modo de presentarse la información y su flujo configuran un importante grado de incertidumbre. Allí es fundamental la flexibilidad de los agentes de salud para responder a necesidades inesperadas de los pacientes. Esas situaciones requieren de mecanismos de tomas de decisiones basados en "la adaptación mutua" (trabajo interdisciplinar, estructuras matriciales), que permiten resolver el problema de salud en el nivel donde se genera la información.

Esta caracterización parece corresponderle a algunas situaciones de trabajo en el CAPS. Podría afirmarse que la numerosidad de procesos, sostenidos a veces simultáneamente, en relación a variados problemas y demandas cuestión constatada en las distintas instancias de observación- condiciona las posibilidades de que la información sea compartida y puesta a trabajar en espacios sistematizados, quedando esta dificultad subsidiada por la participación en ambos equipos de una médica y un psicólogo; quienes trabajan los casos con el equipo, como señaló la médica, de maneras "muy variadas".

Respecto de las dos últimas condiciones -la integración de funciones de dirección o apoyo a las estrategias, y la disposición de los trabajadores al uso de mecanismos de coordinación- restan algunos señalamientos.

En primer lugar, los propios trabajadores manifestaron en las entrevistas la falta de apropiación de algunas herramientas de coordinación, como los instrumentos de registro que podrían ser de uso compartido (el ejemplo más claro son las historias clínicas), y admitieron que el trabajo de intercambio y producción de información respecto de los casos se da de los modos retratados. Podría señalarse que las descripciones dan cuenta de modos de coordinación tendientes a la informalidad, sin sistematización en el uso de herramientas.

Cabe aclarar que los relatos donde hicieron estos señalamientos dan cuenta de una producción cuantiosa así como de logros importantes a partir del trabajo en el espacio grupal, en un escenario cotidiano en el cual sostienen la decisión de realizar prácticas ligadas al paradigma de los derechos humanos, la sustitución de las prácticas médico hegemónicas y manicomiales en los tratamientos dirigidos al sufrimiento psíquico. La complejidad que configura una organización clásica del trabajo, al modo taylorista, la constante tensión con los procesos de atención individual legitimados en tanto prácticas asociadas a las representaciones más tradicionales de la medicina, es parte de la escena diaria de la tarea. Pero es quizás en esa situación a veces caótica, donde parece escabullirse la cuestión de una mayor sistematicidad de las prácticas de coordinación como problema. No se trata de dejar de lado aquí ciertas condiciones de producción de la práctica, la escasa cantidad de horas dedicadas, superposiciones de tareas, etc., pero resulta importante, también, no renegar de la condición de sujeto de los trabajadores.

Durante las entrevistas se han escuchado posiciones y enunciados ligados a distin- 
tas operaciones psíquicas, que podrían ser consideradas mecanismos defensivos frente al sufrimiento en el trabajo: segregación y distanciamiento del otro en tanto diferente; naturalización de la condición de vulnerabilidad del otro; autosuficiencia frente a la tarea; idealización de ciertas herramientas o procedimientos; identificaciones, en relación a imágenes de segregación o soledad; impotencia frente a dificultades; conflictos en torno a procesos de trabajo expresados como rivalidad frente a semejantes; rehusamiento al saber respecto de la implicación frente a determinadas problemáticas. Sería tan aventurado asociar directamente estos fenómenos a determinados efectos o dificultades del trabajo, como desestimar la posibilidad de que éstas se manifiesten de maneras incalculadas en ellos. Puede afirmarse que dan cuenta de procesos de mortificación propios de la vida en institución.

En este punto, se retoma a Onocko (2002) que ubica la cogestión como opción frente a la administración (centralizada y basada en el control) y la autogestión (utópica):

¿Y cómo podríamos otorgar a la gestión un desarrollo de su dimensión creativa?

La primera cuestión sería que instauremos para la gestión un lugar y un tiempo. Crear e instituir espacios en los cuales se pueda experimentar la toma de decisiones colectivas. Espacios en los cuales sea posible formular proyectos.

Espacios que puedan transformarse en instancias, en el sentido de procesar aquello que les llega, y tener un grado mayor de análisis de la implicación con aquello que producen. (p.2)

...Para trabajar en gestión con instancias, es necesario propiciar en esos arreglos otras formas de experimentar la grupalidad... Muchas veces, para tener un proyecto, un grupo precisará de soporte y manejo que lo ayude a estructurarse como intersubjetividad (con un grado razonable de circulación de afectos)...Los procesos transicionales precisan de soporte y de manejo para desenvolverse. (p. 4, 5)

Podemos ubicar una posible dimensión del trabajo de la gestión, en el apoyo de las estrategias, en la instauración de espacios grupales inéditos, para procesar lo que llega - puede agregarse, para elaborar lo vivido y lo pensado. "Trabajando siempre en una tensión transversal, entre lo instituido y lo instituyente, entre el orden que sustenta la operatividad de otros procesos productivos y el desorden de procesos creativos y de cambio" (Onocko, 2002, p.14).

Este modo de pensar la gestión reduce la concentración de poder a las mínimas expresiones necesarias para la construcción y sostenimiento de legalidades. Asimismo, tiende a la democratización interna de la organización sanitaria que, para Testa (1988), "es uno de los eslabones imprescindibles para la transformación del sector salud en un sistema organizativo que se encuentre realmente al servicio del conjunto de la población.” (p.11)

Como ya se ha señalado, la conflictividad propia del campo puede ponerse en juego en forma de tensiones que atraviesan los procesos de trabajo, y resultan del entrecruzamiento de discursos, prácticas, relaciones de fuerza, expresándose de distintos modos: el "para todos" que encierra la universalidad vs. la discriminación presente en las estrategias tendientes a la equidad; tutelaje vs. autonomía en lo referido al cuidado; disciplinamiento vs. legalidad como modos de sostener el transcurrir por el espacio social; normalización vs. 
subjetivación, como ofertas contenidas en distintas propuestas; estrategias colectivas vs. singularidad de los casos.

El dispositivo, en tanto tiene una función que siempre está inscrita en una relación de poder, y resulta del entrecruzamiento de relaciones de poder y de saber, no se reduce al espacio grupal, con sus días y horarios de encuentro, sino que se extiende a todo el reticulado relacional que hace posible su existencia. Por ello puede resultar estratégico como herramienta que permite, mediante el análisis de las condiciones de su institución y existencia, visibilizar y enunciar algunas de esas tensiones.

Esas tensiones no son, en general, impedimentos a salvar, sino conflictos a transitar. Es deseable que ese tránsito sea acompañado de procesos participativos, subjetivantes, en los cuales los trabajadores puedan sostener su decir y revisar su implicación. La posibilidad de desarrollo de esos procesos trazará indicios para conjeturar acerca de qué políticas y qué lazos sociales son impulsados e impulsables por la institución.

Merhy (2006) propone el escenario micropolítico de la gestión del proceso de trabajo en salud bajo la óptica del trabajo vivo, como una manera de analizar la distinción entre el lugar que ese trabajo ocupa para un modelo como el médico hegemónico, y aquel que puede ocupar para otro, como la defensa de la vida.

Es exactamente en ese espacio del trabajo vivo, en el cual los trabajadores de salud reinventan constantemente su autonomía en la producción de actos de salud. Es en ese espacio privado, en que ocurre la relación intersubjetiva entre trabajador y usuario, que se construye y re-construye la libertad de hacer las cosas de manera que produzcan sentido, al menos para los traba- jadores, pero preferentemente para las dos partes. (Merhy, 2006, p.152)

La democratización de la organización, y la producción de espacios colectivos donde analizar y reformular los sentidos de las prácticas -en una interrogación de lo instituido encarnado- pueden ampliar los márgenes de libertad que sostienen al trabajo vivo.

Concomitante, la posibilidad de sostener dispositivos con distribuciones de saber y poder más democráticas, con margen para la creación, la emergencia de lo singular y la producción de autonomía, donde una trabajadora enuncia que "ya pensar que eso existe, que se está moviendo, que hay objetivos y que cada vez crece más, a mí me estimula para trabajar", donde un usuario dice que a partir de su participación en ese y otros espacios se dio cuenta de que "la vida existe, y es algo hermoso".

Existencias que se acoplan, en un camino abierto.

\section{Referencias}

- Agamben, G. (2011). "¿Qué es un dispositivo?” En Sociológica, año 26, número 73 (pp. 249-264). Recuperado el 11 de Noviembre de 2018 de: http://www.scielo. org.mx/pdf/soc/v26n73/v26n73a10.pdf

- Ariel, A. (1994). El estilo y el acto. Buenos Aires: Manantial.

- Cecilio, L. (2009). As Necessidades de Saúde como Conceito Estruturante na Luta pela Integralidade e Eqüidade na Atenção em Saúde. En Roseni Pinheiro, Araujo de Mattos (Eds.), Os sentidos da integralidade na atenção e no cuidado à saúde Rio de Janeiro, Brasil: CEPESC - IMS/UERJ - ABRASCO. Pp. 117-130.

- Ferrandini, D. (2011). Algunos problemas 
complejos de salud. Recuperado el 3 de Marzo de 2019, de http://www.heroinas. net/2013/06/debora-ferrandini.html

- Galende, E. (1998). De un horizonte incierto. Psicoanálisis y Salud Mental en la sociedad actual. Buenos Aires: Paidós.

- Jinkis, J. (1983). Artificio del deseo para conjeturar un estilo. En Conjetural, Revista psicoanalítica. Número 1. Buenos Aires: Sitio. Pp. 9-22.

- Merhy, E. (2013) En busca del tiempo perdido: La micropolítica del trabajo vivo en salud. En Franco, T. y Merhy, E. (Eds.) Trabajo, producción del cuidado y subjetividad en salud. Buenos Aires: Lugar. Pp. 19-60.

- Merhy, E., Feuerwerker, L., Burg Ceccim, R. (2006). Educación Permanente en Salud: una Estrategia para Intervenir en la Micropolítica del Trabajo en Salud. En Salud Colectiva. Volumen 2, Número 2. Buenos Aires. Pp. 147-160.

- Onocko Campos, R. (2002). La gestión: espacio de intervención, análisis y especificidades técnicas. Recuperado el 11 de Mayo de 2018, de https://psicolog.org/ la-gestin-espacio-de-intervencin-anlisis-y-especificidades-tcn.html

- Organización Mundial de la Salud (1978). Declaración de Alma-Ata. Conferencia Internacional sobre Atención Primaria de Salud, Alma-Ata. Recuperado de: https:// www.paho.org/hq/dmdocuments/2012/ Alma-Ata-1978Declaracion.pdf

- Percia, M. (1997). Notas para pensar lo grupal. Buenos Aires: Lugar

- Rovere, M. (2002). La Planificación Estratégica y la Ciudad como Sujeto. Recuperado el 27 de Marzo de 2019, de https:/ / www.google.com/url?sa=t\&rc$\mathrm{t}=\mathrm{j} \& \mathrm{q}=\& \mathrm{esrc}=\mathrm{s} \&$ source $=$ web\&cd $=\&-$ ved=2ahUKEwjZpaPT_aXrAhUQHrkGHUKDBckQFjABegQICxAD\&url=https $\% 3 \mathrm{~A} \% 2 \mathrm{~F} \% 2$ Felagoraasociacioncivil. files.wordpress.com $\% 2 \mathrm{~F} 2013 \% 2 \mathrm{~F} 08 \%$ 2Fla-planificacic3b3n-estratc3a9gica-yla-cuidad-como-sujeto.pdf\&usg $=\mathrm{AOv}$ Vaw1rzkmBvqtTYCvTsflBNs-_

- Sávio Alves, D. (2009). Integralidade nas Políticas de Saúde Mental. En Roseni Pinheiro, Araujo de Mattos (Eds.). Os sentidos da integralidade na atenção e no cuidado à saúde Rio de Janeiro, Brasil: CEPESC - IMS/ UERJ - ABRASCO. Pp.171-180.

- Sousa Campos, G. (2001). Gestión en salud. En defensa de la vida. Buenos Aires: Lugar.

- Terraza Núñez, R., Vargas Lorenzo, I. y Vázquez Navarrete, M. (2006). La coordinación entre niveles asistenciales: una sistematización de sus instrumentos y medidas. Gaceta Sanitaria, 20 (6). Pp. 485-495. Recuperadoen 31 dejulio de2020, dehttp:/ / scielo.isciii.es/scielo.php?script $=$ sci_arttext\&pid=S0213-91112006000600012\&1ng=es\&tlng=es.

- Testa, M. (1988). Atención iprimaria o primitiva? de salud. Recuperado el 10 de Marzo de 2019 de: http://www.trabajosocial. unlp.edu.ar/uploads/docs/testa__mario__atencion_primaria_o_primitiva_ de_salud.pdf

- Ulloa, F. (2011). Novela Clínica Psicoanalítica. Buenos Aires: Libros del Zorzal.

- Vargas, I., Vázquez, M., Henao, D., Campos, C. (2009). Implantación de mecanismos de coordinación asistencial en redes integradas de servicios de salud (RISS). Recuperado el 7 de abril de 2018, de https://www.google.com/url?sa=t\&rct=$\mathrm{j} \& \mathrm{q}=\&$ e s r c $=$ s $\&$ s o u r c e $=$ web $\&$ c $\mathrm{d}=15$ \&ved $=2$ ahUKEwi66J2s0ajgAhXuE 7 k G H d g 8 B r s Q F j A e g Q I A hAC\&url=http $\% 3 \mathrm{~A} \% 2 \mathrm{~F} \% 2 \mathrm{Fwww}$. equity-la.eu $\% 2$ Fupload $\% 2$ Farxius $\%$ 2F20160914094910-Vargas\%25202009_ Guia\%2520mecanismos\%2520de\%2520coordinacion.doc\&usg $=\mathrm{AOvVaw} 3 \mathrm{xkp}-$ 
3Di0zWNC67CcesMc2I

- Winnicott, D. (2007). Realidady Juego. Buenos Aires: Gedisa.

- Zaldúa, G., Pawlowicz, M., Tisera, A., Longo, R., Sopransi, M., Lenta, M., Moschella, R. y Lohigorry, J. (2014). Obstáculos y posibilidades de la implementación de la ley Nacional de Salud Mental: un estudio comparativo entre 2010 y 2013. Recuperado el 10 de julio de 2019, de https: / /www. aacademica.org /maria.pia.pawlowicz/52. pdf 